\title{
Neutralization Processing and Study of Dynamic of Acidic Tailings Used Heap Leaching
}

\author{
Meng Shu, Zhong Pingru, Wu Yongyong \\ Beijing Research Institute of Chemical Engineering and Metallurgy \\ Jiukeshu No.145 Tongzhou District Beijing, Beijing, China \\ ygy@bricem.com.cn
}

\begin{abstract}
In this study, a newly dumped uranium tailings used acid heap-leaching from China was treated with calcium and sodium hydroxide as the neutralizer to perform a neutralised experiment in order to exam the trend of $\mathrm{pH}$ along with period and simulate the kinetic equations. Combined with the distribution of size, the porosity and the pore volume of waste ore, the reasons that affect the $\mathrm{pH}$ trend of the neutralization system of uranium heap-leaching waste were pointed out. The result showed the final $\mathrm{pH}$ of tailings system rise with the use amount of neutralizers, while the over doze of neutralizer tend to disturb the system. Both Lime and sodium hydroxide were considered to be effective in neutralization.
\end{abstract}

Keywords: Uranium, Heap-Leaching Tailings, Neutralization, Porosity.

\section{Introduction}

In China, acid heap leaching technology has been used increasingly in hard rock uranium mines. On the other hand, the resulting heap leaching tailings acidic waste water, not only affect the sewage discharging standard, also bring a series of problems to mine geological conditions, land reclamation, etc. To solve this problem, currently the widely used disposal process for acidic heap leaching tailings is to add lime into the tailings with sufficiently mixing, then permanent stored in tailings repositories ${ }^{[1,2]}$. However, while being long-time piled up in repositories, the already neutralized tailings often go back to acid nature with the phenomenon that the tailings seepage water $\mathrm{pH}$ value gradually reduce, finally from neutral to acid, leading the formation of acidic mining waste water (AMD). This kind of phenomenon is not rare in acid uranium mine tailings ponds ${ }^{[3]}$. Recent years, with the great development in uranium mining and expanding of heap leaching uranium mine enterprises, the amount of acid tailings and acidic water seepage continue to increase. With this kind of phenomenon getting more and more serious, the cost of production for the uranium mine would be too large due to the extra acidic water seepage process and increasing wastewater treatment capacity, needless to say the pollution to the water, soil and environment around the tailing dams.

In this study, the acid heap leaching tailing of a uranium mine in south China was testified with all sorts of methods. First, the microscopic textures and the parameters of tailings were tested. During the neutralizing process, influences of various microscopic physical properties were analyzed. Moreover, in laboratory, different neutralizers were testified to react with tailings and the effect of neutralization over time was observed. Through the linear fitting of the Ph change curve and the creation of neutral dynamic equation, the neutralization characteristics of tailings, the relationship between $\mathrm{pH}$ and reaction time and the proper dosage of neutralizer were further judged.

\section{Materials and Methods}

\subsection{Materials}

A newly dumped tailings from a uranium deposit using heap leaching in Jiangxi province before the neutralization treatment was used as the raw material in the experiments. The particle size of the material was $-8 \mathrm{~mm}$, the starting $\mathrm{pH}$ around 4, the uranium grade $0.004 \%$.

Two types of reagents were used for neutralization in the experiments, including industrial lime with $\mathrm{CaO}$ purity of $74.1 \%$ and $\mathrm{AR} \mathrm{NaOH}$ with purity of $97 \%$. Pure water was used as binder liquid (surface tension $0.072 \mathrm{~N} / \mathrm{m}$ ). 


\subsection{Equipment}

In all experiments, the acidity meter was used to measure the $\mathrm{pH}$ of the tailing system and neutralization process, witch was a ray magnetic brand $\mathrm{ph}-3 \mathrm{f} \mathrm{pH}$ meter and made by Shanghai precision instrument company, with the $\mathrm{pH}$ range from 0 to 14.

The microscopic parameters were carried out by the physical adsorption instrument (Tristar of British Mike Instruments Company).

\subsection{Measurements of Particle Size Distribution}

To study the different mechanisms of tailing properties and behaviors during the neutralization process, different experiments were performed.

In the experiment of particle size distribution measurement, first the dry tailing sample was weighted. Then according to take $100 \mathrm{~g}$ tailing samples, with a $0.165 \mathrm{~mm}, 0.495 \mathrm{~mm}$ and $1.25 \mathrm{~mm}, 4 \mathrm{~mm}$ to wet sieve, sieve, in turn, will use the proceeds the particle size of tailings drying to conservation of mass, record results, after drying the total mass loss $<0.1 \%$.

\subsection{Porosity and Surface Area Measurement}

Tailings particle size samples will be $2 \mathrm{~h}$ under $110{ }^{\circ} \mathrm{C}$ drying, apply adequate amount in the sample tube $(0.165 \mathrm{~mm}$ samples by 2 mpa pressure), the sample under the vacuum degassing heating; Using nitrogen as gas adsorption, helium as stripping gas, the determination of samples with different grained tailings of low temperature nitrogen isothermal adsorption stripping, using BET equation to calculate the sample specific surface area and entrance, according to the grade distribution and entrance to calculate the porosity of the sample.

\subsection{Neutralization Test}

The neutralization test is carried out in the beaker, and it is used in the soaking mode, and the tail residue sample and water is added to the solid quality ratio of 1 to 1 . Lime and sodium hydroxide were used as the neutralizing agent and the beaker was added. Lime was added to the residue quality of $0.4 \%, 0.5 \%, 0.6 \%, 0.7 \%, 0.8 \%$ and $0.9 \%$, respectively, and sodium hydroxide was added at $0.4 \%, 0.45 \%, 0.5 \%, 0.6 \%, 0.7 \%$ and $0.8 \%$ respectively. A blank sample was established, that is, only clear water and no neutralizer in the tail slag sample. The $\mathrm{pH}$ value of each beaker is recorded daily and the amount of water lost by natural factors such as evaporation is added according to the weight of each sample at the beginning of the test.

\section{Results and Discussion}

\subsection{Slag Granulation Distribution and Texture Characterization}

The particle size distribution and the texture properties of the tail slag samples were shown in TAB. 1 .

Table 1: Granule Distribution and Texture Properties of Heap Leaching Residue.

\begin{tabular}{|c|c|c|c|c|c|}
\hline Particle Size & $-0.165 \mathrm{~mm}$ & $0.165 \mathrm{~mm} \sim 0.495 \mathrm{~mm}$ & $0.495 \mathrm{~mm} \sim 1.25 \mathrm{~mm}$ & $1.25 \mathrm{~mm} \sim 4 \mathrm{~mm}$ & $+4 \mathrm{~mm}$ \\
\hline $\begin{array}{c}\text { Mass } \\
\text { Fraction/\% }\end{array}$ & 7.74 & 13.31 & 35.67 & 23.16 & 20.11 \\
\hline $\begin{array}{c}\text { Pore Volume } \\
/ \mathrm{cm}^{3} \cdot \mathrm{g}^{-1}\end{array}$ & 0.037 & 0.013 & 0.010 & 0.008 & 0.008 \\
\hline $\begin{array}{c}\text { Specific } \\
\text { Surface Area/ } \\
\mathrm{m}^{2} \cdot \mathrm{g}^{-1}\end{array}$ & 23.047 & 8.783 & 7.011 & 4.601 & 6.581 \\
\hline
\end{tabular}

From the result of graded distribution of the tailings from the table, it is obvious that the heap leaching ore grade distribution range is larg. Also that the coarse-grained tailing particle accounts for a bigger mass fraction, witch is relivent to the requirement a good permeability during heap leaching process. In fact, because of this special properties, tailings 
library gap can reach $40 \% \sim 60 \%$ in the tailings storage ${ }^{[4]}$. Moreover, most of heap the acid residue in leaching tailings exist in large tailing particles, on the surface of the concave, convex and wrinkles. When the neutralizer was added, this part of the residual acid would be react with neutralizing reagent first.

For the pore of the hard rock ore itself is quite small, two reasons causes of pores in tailings. One is during the process of crushing, external force produced inside deformating the pore and fissure of ore particle. And the second reason is during heap leaching, due to the erosion of leaching agent in the mineral surface and internal of ore particle ${ }^{[5]}$. According to the results of the pore volume TAB. 1, the pore volume of heap leaching residue has a tendency to decrease with the increase of the particle size. The reason is in the process of crushing, the small ore particle faces a larger direction, often leading to a larger internal deformation is larger, resulting in larger pores in the ore. Based on the weighted average calculation of the results of the pore volume and the size distribution, the total porosity of the sample heap leaching tailing was $2.88 \%$, the volume of the pore in the tailings $37.21 \mathrm{~L} / \mathrm{t}$. In heap leaching operation, the acidity of leaching agent generally per litter a dozen to a few grams. At the end of the heap leaching leaching cycle, each ton of heap leaching tailings internal may contain more than dozens to hundreds of grams acid. This part of the residual acid cannot react with neutralizer at the first period, but within a long period of weathering or rain erosion, through concentration and capillary action, or under the osmosis of neutralizer, gradually be burned off.

From the specific surface area of each fraction distribution result in TAB.1, it can be seen that the specific surface area of the heap leaching tailings is large, with $0.165 \mathrm{~mm}$ tailings of specific surface area reached more than $20 \mathrm{~m}^{2} / \mathrm{g}$. The specific surface area decreases with increasing of tailings particle size trends, witch is due to the small tailings particles have larger pore volume than large particles. For particle greater than $1 \mathrm{~mm}$, the surface area has not decreased since the pore volume has not changed greatly. The neutralization reaction happened inside the tailings can be explained by two kinds of fluid flow in tailings pore, diffusion, and the process of a series of chemical reactions. One is the fluid of neutralizer. The other is the fluid of acid exist on the surface or inside of the ore particle ${ }^{[6,7]}$. As a result, the larger the surface area, the greater the contact area were between the neutralizing agent and the amount of acid in the pore. For larger particles with relatively small surface area, due to the small amount of contact area, the time of neutralization reaction is increased to a certain extent.

\subsection{Neutralization Curve}

Two kinds of strong alkaline neutralizers were selected and their neutralization effect was investigated. The alkalinity of sodium hydroxide is the strongest, which can immediately react with the residual acid on the surface of the residue or outflow to reflect the change of $\mathrm{pH}$ in the neutralization system. The neutralization reaction of lime as a common use in the slag of mine tailings can be used to simulate the actual situation in tailing.

According to the neutralization test and the change of $\mathrm{pH}$ value over time, the curve fitting for each group was given. The fitting curves of test data of each group are shown in FIG.1 and FIG. 2:

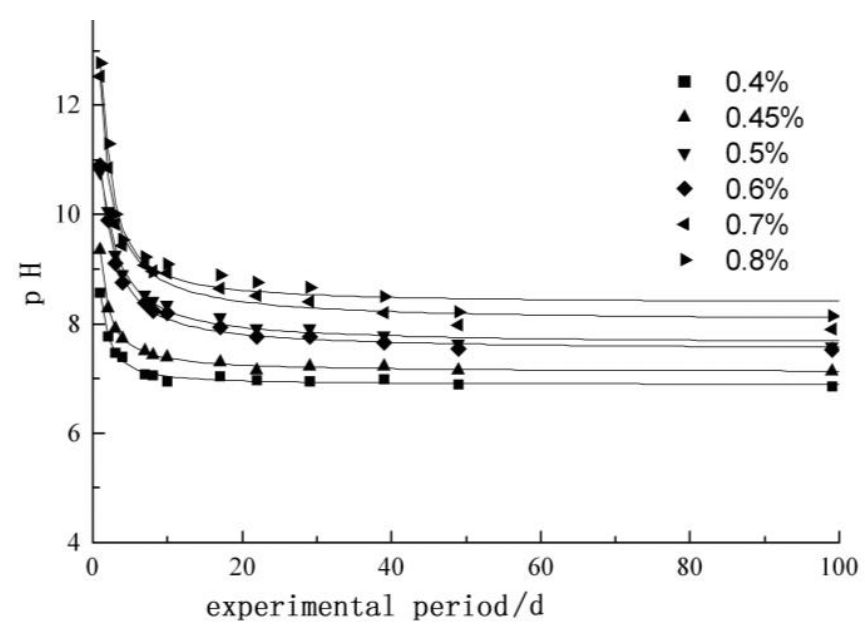

Fig. 1: Neutralization curve of tailing with different addition ratio of $\mathrm{NaOH}$. 


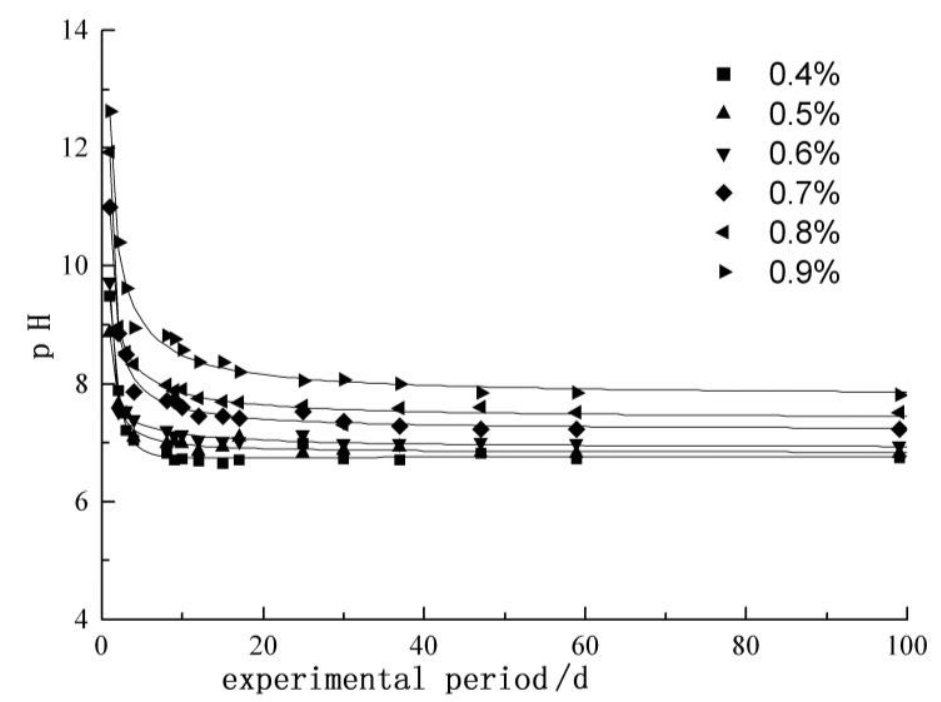

Fig. 2: Neutralization curve of tailing with different addition ratio of lime.

Before neutralizing, the $\mathrm{pH}$ value of the system remain between 3 to 4 , notably acidic. In some cases, system pH vakye would experienced a slowly rising because of dissolvation of the residual acid into water or physical reactions such as calcium, magnesium. By two results seen in the picture, by adding the neutralizer, the $\mathrm{pH}$ value of the system decreased rapidly in the initial 2 to $3 \mathrm{ds}$. After that, the rate of decrease gradually slows down to a relatively stable stage, with the $\mathrm{pH}$ value of each beaker in this interval slightly fluctuating. The $\mathrm{pH}$ of the system stabilizes within about 20ds after neutralization. The final $\mathrm{pH}$ value of neutralization system is stable in the range of 6 to 9 , which is in line with the emission standard of mine process wastewater. Compared with figure 1 and figure 2, it can be found that the difference of neutralization effect using different neutralizer and the variation of $\mathrm{pH}$ value of the neutralization system are not significant in the same neutralization dosage.

\subsection{Neutralization Dynamics Model}

The kinetic equations were simulated according to the fitting curve obtained from the residue neutralization test. Represented as follows:

$$
\begin{array}{lll}
\mathrm{NaOH} \text { addition 0.4\%: } & y=6.88+\frac{1.59}{t}+\frac{0.74}{t^{2}}-\frac{0.64}{t^{3}} & \mathrm{R}=0.986 \\
\mathrm{NaOH} \text { addition } 0.45 \%: & y=7.12+\frac{2.67}{t}-\frac{0.85}{t^{2}}+\frac{0.41}{t^{3}} & \mathrm{R}=0.997 \\
\mathrm{NaOH} \text { addition 0.5\%: } & y=7.52+\frac{6.03}{t}-\frac{3.00}{t^{2}}+\frac{0.36}{t^{3}} & \mathrm{R}=0.994 \\
\mathrm{NaOH} \text { addition } 0.6 \%: & y=7.62+\frac{6.61}{t}-\frac{4.18}{t^{2}}+\frac{0.74}{t^{3}} & \mathrm{R}=0.988 \\
\mathrm{NaOH} \text { addition } 0.7 \%: & y=8.04+\frac{7.75}{t}-\frac{6.55}{t^{2}}+\frac{3.29}{t^{3}} & \mathrm{R}=0.981
\end{array}
$$




$$
\begin{aligned}
& \mathrm{NaOH} \text { addition } 0.8 \%: \quad y=8.36+\frac{4.90}{t}+\frac{3.13}{t^{2}}-\frac{3.61}{t^{3}} \quad \mathrm{R}=0.975 \\
& \mathrm{Ca}(\mathrm{OH}) 2 \text { addition } 0.4 \%: \quad y=6.77-\frac{1.51}{t}+\frac{10.74}{t^{2}}-\frac{6.52}{t^{3}} \quad \mathrm{R}=0.965 \\
& \mathrm{Ca}(\mathrm{OH}) 2 \text { addition } 0.5 \%: \quad y=6.83+\frac{1.37}{t}+\frac{0.63}{t^{2}}+\frac{0.04}{t^{3}} \quad \mathrm{R}=0.974 \\
& \mathrm{Ca}(\mathrm{OH}) 2 \text { addition } 0.6 \%: \quad y=6.91+\frac{3.04}{t}-\frac{6.48}{t^{2}}+\frac{6.25}{t^{3}} \quad \mathrm{R}=0.990 \\
& \mathrm{Ca}(\mathrm{OH}) 2 \text { addition } 0.7 \%: \quad y=7.18+\frac{4.74}{t}-\frac{4.38}{t^{2}}+\frac{3.46}{t^{3}} \quad \mathrm{R}=0.995 \\
& \mathrm{Ca}(\mathrm{OH}) 2 \text { addition } 0.8 \%: \quad y=7.41+\frac{4.33}{t}-\frac{4.99}{t^{2}}+\frac{5.18}{t^{3}} \quad \mathrm{R}=0.992 \\
& \mathrm{Ca}(\mathrm{OH}) 2 \text { addition } 0.9 \%: \quad y=7.79+\frac{7.72}{t}-\frac{8.10}{t^{2}}+\frac{5.22}{t^{3}} \quad \mathrm{R}=0.986
\end{aligned}
$$

Using the common characteristics of the above equation, all the equations can be reduced to a general formula:

$$
y=a+\frac{b}{t}+\frac{c}{t^{2}}+\frac{d}{t^{3}}
$$

Definition of the parameters in the equation can be separately as follows: Parameter $a$ is the ultimate stability of the neutral system $\mathrm{pH}$ value, namely that the $\mathrm{pH}$ value of tailings and would be infinitely approach to $a$, while keeping it in a very small amplitude area. The value of $a$ depends on the neutralization agent dose. After going through an initial rapid decline with the addition of alkaline, the $\mathrm{pH}$ value of neutralization reaction system would still keep a nonlinear oscillation in the mid and late term of response. The main factor is neutralization reaction happened between the neutralizer and acid in the pore of residual under the long time infiltration. Other factors include the influence of environment, temperature, ion concentration and diffusion in solution. Parameters $b, c$ and $d$ can be characterized by all the external factors witch cause the initial $\mathrm{pH}$ decrease. It can be seen from the equation that the influence of external factors on the stability of the neutralization system is getting smaller and smaller as time goes by.

The $\mathrm{R}$ value in the equation also reflects the oscillation amplitude of neutralization system to some extent, which is closely related to the stability of the system. The closer the $\mathrm{R}$ value is to 1 , the smaller the oscillation of the neutralization system. In neutral system, therefore, not only should choose appropriate for the amount of neutralizer, making the ultimate neutral $\mathrm{pH}$ conform to the requirements, also pay attention to the different amount of neutralizer to join and system oscillation amplitude. A small oscillation amplitude system should be selected as far as possible, to maintain the system during and after the period of stability.

From the above dynamics equation of tailings, it can be speculated that when using lime and sodium hydroxide as a neutralizer, the stability of the final $\mathrm{pH}$ value (that is, a value) increased with increasing along with the use of alkaline. While the $\mathrm{R}$ value increased at first, then fall. According to the standards of waste water discharge, in theory, to join in the heap leaching tailings samples of the selected base of tailings quality between $0.4 \%$ and $0.9 \%$, can meet the requirement and processing, namely after neutralization processing tailings $\mathrm{pH}$ neutralization system between $6 \sim 9$. But according to different neutralizer add quantity under the condition of $\mathrm{R}$ value comparison, in the amount of $\mathrm{NaOH}$ is $0.8 \%$ and the amount of lime is $0.8 \%$, the $\mathrm{R}$ values fall instead, explaining that in this situation, the neutralizer is overused and would lead to a decrease of the system stability. 


\section{Conclusions}

1) It could be divided into two stages in the neutralization reaction of heap leaching residue. In the first phase, the neutralizing agent and the surface acid were neutralized, and the reaction time was only a few days, while the $\mathrm{pH}$ value in the interval and the system decreased rapidly. In the second stage, the reaction of the neutralizing agent and acidic substance happened inside the tailings pore, thus resulting a long reaction time and a relatively slowly $\mathrm{pH}$ value decrease, sometimes accompanied with repeated fluctuations.

2) The dynamics equations according to the $\mathrm{pH}$ change over time was a better way to simulate the tailings neutralization system and reaction process, namely: the final $\mathrm{pH}$ of neutralization system is made up by the amount of neutralizer as the main influence factors as well as the microscopic texture and tailings by themselves and other external factors such as joint decisions. Moreover, the $\mathrm{pH}$ value of the endpoint increased with the addition of neutralization agent. However, the $\mathrm{pH}$ stability of the neutralization system decreased when the quantity of the mixture is too high.

3) The neutralization effect of lime in the experiment is comparable with that of sodium hydroxide. With a reasonable use doze, the system could be neutralized well and stable. Thus, lime would be the ideal neutralization reagent for the disposal of the tailings residue compared to sodium hydroxide considering the economic cost. The neutralization process of the acid heap leaching residue of mine could be investigated through the combination of neutralization test of tail slag in the test chamber and the simulation of kinetics. In this way, this method is appropriate to determine the suitable tail-slag and alkali content to solve the problem of acid recovery of slag in the slag.

\section{References}

[1] P. Li, "Feasibility of Using Treated Remains From Uranium Ore Leaching Heap As Backfill Material For Underground Stopes," J. China Mining Magazine, 2002, vol. 11, no. 4, pp. 64-65.

[2] P. Zhong, T. Li, Y. Mao, S. Li, "Uranium Ore Treatment By Percolation Leaching At Fuzhou Utanium Mine," J. Uranium Mining and Metallurgy, vol. 23, no. 1, pp. 13-17, 2004.

[3] J. Jurjovec, C. J. Ptacek, D. W. Blowes, "Acid neutralization mechanisms and metal release in mine tailings: A laboratorycolumn experiment," J. Geochimica et Cosmochimica Acta, vol. 66, no. 9, pp.1511-1523, 2002.

[4] M. Zhang, "Leaching Technology," M Beijing, Atomic Energy Press, 7-5022-1078-4, 1994.

[5] C. Li, W. Zhou, "Simulation Test of Hydraulic Filling With Tailings of Neutralization Heap Leaching Tail," $J$. Industrial Minerals \& Processing, vol. 24, no. 3, pp. 14-17, 1995.

[6] G. Daccord, O. Lietard, R. Lenormand. Chemical Dissolution of a Porous Medium by a Reactive Fluid-II. Convection vs Reaction," J Chemical Engineering Science, vol. 48, no. 1, pp. 179-186, 1993.

[7] Q. Kang, D. Zhang, S. Chen, "Simulation of dissolution and precipitation inporous media," J. Journal of Geophysical Research, vol. 108, no. 10, pp. 1-8, 2003.

[8] G. Daccord, "Chemical Dissolution of a Porous Medium by a Reactive Fluid," J Physical Review Letters, vol. 58, no. 5, pp. 479-482, 1987. 